\title{
ЭЛЕКТРОННЫЙ ПАРАМАГНИТНЫЙ РЕЗОНАНС ХРОМА В СУЛЬФИДЕ КАЛЬЦИЯ
}

Исследованы спектры электронного парамагнитного резонанса (ЭПР) ионов $\mathrm{Cr}^{3+}$ и $\mathrm{Cr}^{+}$в поликристаллическом $\mathrm{CaS}$. Малая ширина линий поглощения и отсутствие начального расщепления основного уровня ${ }^{4} F_{3 / 2}$ свидетельствуют о том, что ионы $\mathrm{Cr}^{3+}$ расположены в электрическом поле октаэдрической симметрии. Значения $g$-фактора и константы $A$ сверхтонкой структуры (CTC) хромовых центров в $\mathrm{CaS}$ близки к значениям соответствующих параметров $\mathrm{Cr}^{3+}$ и $\mathrm{Cr}^{+}$в других соединениях $\mathrm{A}_{\mathrm{II}} \mathrm{B}_{\mathrm{V}}$. Изучена термическая стабильность центров хрома в $\mathrm{CaS}$. Ионы $\mathrm{Cr}^{3+}$ и $\mathrm{Cr}^{+}$могут быть использованы в качестве реперных центров при определении знака рекомбинационных процессов в $\mathrm{CaS}$.

\section{1. Введение}

K настоящему времени проведено сравнительно много исследований, посвященных парамагнитным свойствам хрома в разных веществвах. $\mathrm{B}$ соединения $\mathrm{A}_{I I} \mathrm{~B}_{V I}$ наиболее изученными являются ионы хрома в зарядовом состоянии $\mathrm{Cr}^{3+}$ и $\mathrm{Cr}^{+}$. В значительно меньшей мере исследован ЭПР ионов $\mathrm{Cr}^{2+}$ (см., напр., обзор [ $\left.\left.{ }^{1}\right]\right)$. Тем не менее надежно установлено, что в соединениях $\mathrm{A}_{\mathrm{II}} \mathrm{B}_{\mathrm{VI}}$, для которых характерны сравнительно большая степень ковалентности химической связи и тетраэдрическое расположение ионов, хром замещает катионы основания и обладает зарядовым состоянием $\mathrm{Cr}^{2+}$. При возбуждении таких кристаллов ионизирующей радиацией $\mathrm{Cr}^{2+}$ захватывает электрон и переходит в $\mathrm{Cr}^{+}$, который легко обнаруживается и идентифицируется методом ЭПР $\left[{ }^{2-4}\right]$. В указанных кристаллах $(\mathrm{ZnS}, \mathrm{ZnSe}, \mathrm{ZnTe}, \mathrm{CdS})$ ионы $\mathrm{Cr}^{3+}$ не обнаружены.

Трехвалентное состояние свойственно ионам хрома в кристаллах с кубической симметрией кристаллической решетки и большой степенью ионности связи. ӘПР ионов $\mathrm{Cr}^{3+}$ в $\mathrm{MgO}$ детально исследовался в $\left[{ }^{5-7}\right]$. Ионы $\mathrm{Cr}^{3+}$ обнаружены также в $\mathrm{CaO}$ и $\mathrm{MgS}\left[{ }^{8}\right]$. О наличии $\mathrm{Cr}^{+}$в таких матрицах известно только сообщение $\left[{ }^{9}\right]$.

$\mathrm{CaS}$ кристаллизуется в решетку типа $\mathrm{NaCl}$ и обладает смешанным типом связи, занимая по степени ионности промежуточное положение между $\mathrm{ZnS}$ и $\mathrm{MgO}$. В настоящей работе методом ЭПР установлено, что хром встраивается в решетку $\mathrm{CaS}$ в виде трехзарядных ионов. В определенных условиях, например в процессе рентгеновского облучения закаленных образцов $\mathrm{CaS}-\mathrm{Cr}$, может возникать состояние $\mathrm{Cr}^{+}$, причем исходным состоянием служит $\mathrm{Cr}^{2+}$. 


\section{2. Методика эксперимента}

Исследовался ЭПР поликристаллических образцов $\mathrm{CaS}$ как активированных, так и неактивированных ионами хрома (в последнем случае хром содержался в $\mathrm{CaS}$ в виде неконтролируемой примеси). При изготовлении фосфоров использовался $\mathrm{CaS}$ с фазовой чистотой $95-98 \%$. Активатор вводился в виде $\mathrm{Cr}\left(\mathrm{NO}_{3}\right)_{3}$ путем прокаливания смеси $\mathrm{CaS}$ и соли хрома в атмосфере серы. Время и температура прокаливания варьировались в пределах $1-2$ ч и $1000-1100^{\circ} \mathrm{C}$ соответственно. Спектры ЭПР регистрировались спектрометром РЭ 1301. Для облучения объектов рентгеновскими лучами использовалась установка УСР-55. Все измерения осуществлялись при температуре жидкого азота. Напряженность магнитного поля определялась измерителем магнитной индукции ИМИ-2.

\section{3. Экспериментальные результаты}

Ионы $\mathrm{Cr}^{3+}$ в CaS. Спектр ЭПР хрома в $\mathrm{CaS}$ показан на рис. 1, a. Он состоит из центральной линии с $g=1,9831 \pm 0,0005$ и квартета линий СТС с интенсивностью компонент, составляющей $1 / 42 \pm 5 \%$ от интенсивности центральной линии. Расстояния между линиями квартета приблизительно равны между собой $(\Delta H=17,2 \pm 0,1 \Gamma c)$ и слегка увеличиваются с ростом напряженности магнитного поля. По нашему мнению, описанный выше спектр обусловлен ионами $\mathrm{Cr}^{3+}$, замещающими $\mathrm{Ca}^{2+}$ в $\mathrm{CaS}$. $\mathrm{Cr}^{3+}$ имеет электронную конфигурацию $3 d^{3}$ и основной терм ${ }^{4} F_{3} / 2$. В октаэдрическом поле семикратно вырожденный уровень расщепляется на орбитальный синглет ${ }^{4} A_{2 g}$ и два высоколежащих триплета ${ }^{4} T_{2 g}$ и $T_{1 g}$. Основное состояние, ответственное за парамагнитный резонанс ${ }^{4} A_{2 g}$, удалено от ближайшего возбужденного состояния ${ }^{4} T_{2 g}$ на

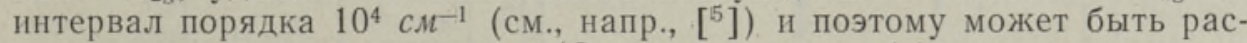
смотрено как изолированное ${ }^{4} S$ состояние с эффективным спином $\widetilde{S}=3 / 2$. Во внешнем магнитном поле вырождение по спину снимается, и между четырьмя подуровнями с разными $m_{S}$ могут быть индуцированы три перехода, подчиняющиеся правилам отбора $\Delta m_{\mathrm{S}}= \pm 1$.

Если симметрия октаэдрического поля не нарушена, то энергии этих переходов совпадают и в спектре ЭПР наблюдается только одна линия поглощения. В спектре, изображенном на рис. 1, $a$, это интенсивный сигнал с изотропным g-фактором, равным 1,9831. СТС обусловлена взаимодействием между ядерным магнитным моментом изотопа ${ }^{53} \mathrm{Cr}^{3+}$ $(9,55 \%, I=3 / 2)$ и сверхтонким магнитным полем на ядре ${ }^{53} \mathrm{Cr}^{3+}$. Отношение интенсивности центрального сигнала от центров с четными изотопами ${ }^{50} \mathrm{Cr},{ }^{52} \mathrm{Cr}$ и ${ }^{54} \mathrm{Cr}(90,45 \%, I=0)$ к интенсивности линий СТС, вычисленное на основе процентного содержания изотопов в природном хроме, немного меньше, чем $1 / 40$. Эта величина хорошо согласуется с величиной $1 / 42 \pm 5 \%$, определенной из эксперимента. Положение линий спектра ӘПР иона $\mathrm{Cr}^{3+}$ в $\mathrm{CaS}$ может быть описано спин-гамильтонианом

$$
H=g \beta \hat{\mathbf{H}} \hat{\mathbf{S}}+A \hat{\mathbf{I S}},
$$

где $S=3 / 2$ и $I=3 / 2$. Диагонализация соотношения (1) с учетом членов до второго порядка теории возмущений дает следующее выражение для уровней энергии:

$$
E_{m_{s} m_{I}}=g \beta H m_{\mathrm{S}}+A m_{\mathrm{S}} m_{I}+
$$




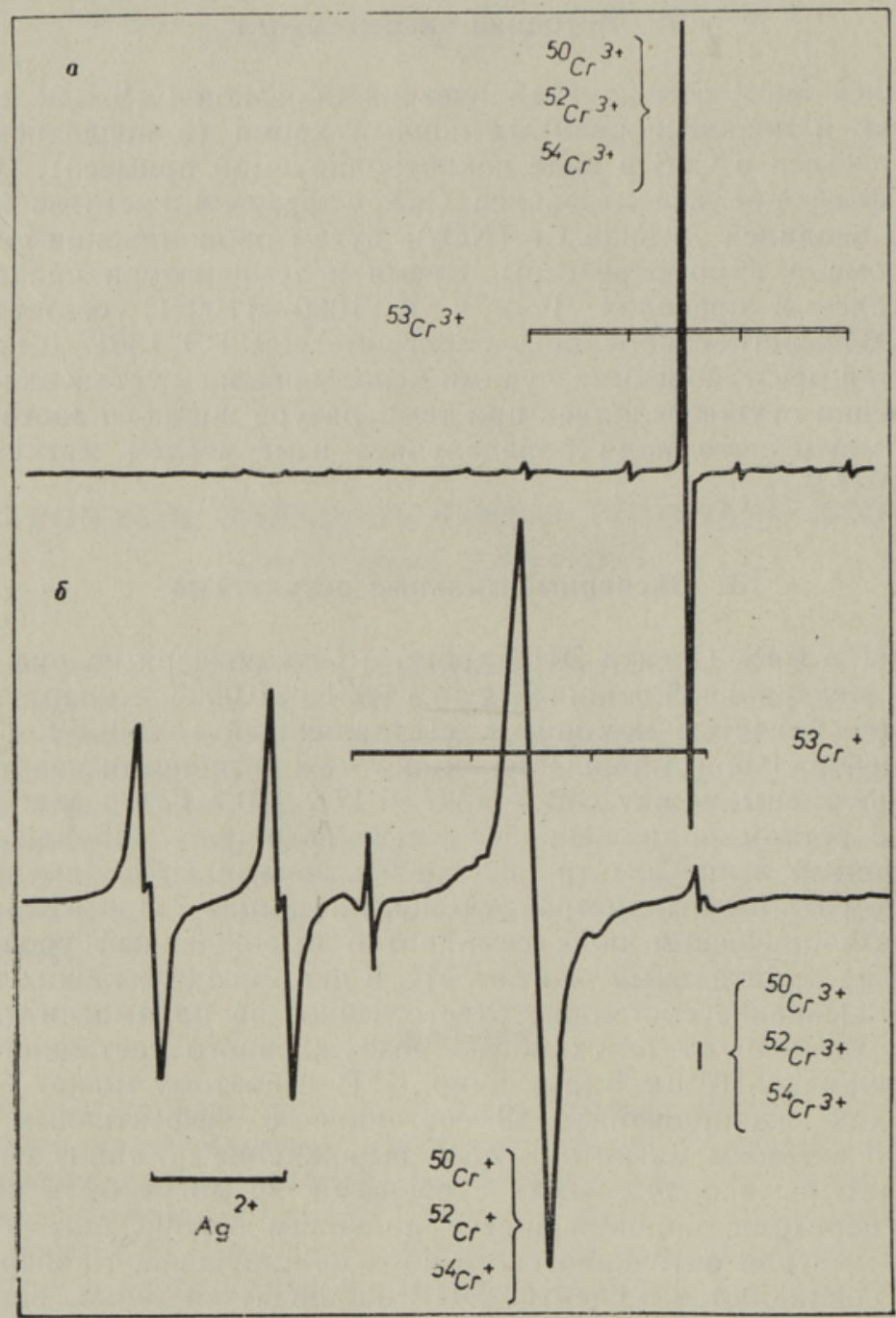

Рис. 1. Спектры ЭПР хрома в $\mathrm{CaS}: a-$ ионов $\mathrm{Cr}^{3+}$ в закаленном фосфоре $\mathrm{CaS}-\mathrm{Cr}, \mathrm{Ag} ; 6$ - того же фосфора после облучения рентгеновскими лучами при $298 \mathrm{~K}$.

$$
+\frac{A^{2}}{2 g \beta H}\left[I(I+1) m_{S}-S(S+1) m_{I}+m_{S} m_{I}\left(m_{S}-m_{I}\right)\right] .
$$

Отсюда получаем условие резонанса для разрешенных переходов $\left(m_{S}, m_{I}\right) \rightarrow\left(m_{S-1}, m_{I}\right)$ при постоянной частоте спектрометра $v:$

$$
g \beta H=h v-A m_{I}-\frac{A^{2}}{2 h v}\left[I(I+1)+m_{I}\left(2 m_{S}-m_{I}-1\right)\right],
$$

где $h-$ постоянная Планка и $\beta$ - магнетон Бора. Выражение, пропорциональное $A^{2}$, описывает "незначительную неэквидистантность $(\approx 0,5 \%)$ линий СТС.

Подстановка в уравнение (2) найденных из эксперимента значений 
частоты и магнитных полей, при которых наблюдаются соответствующие резонансные переходы, позволяет определить $g$ и $A$ иона $\mathrm{Cr}^{3+}$ в $\mathrm{CaS}$. Эти значения, наряду с известными параметрами ионов $\mathrm{Cr}^{3+}$ и $\mathrm{Cr}^{+}$ в некоторых других соединениях $\mathrm{A}_{I I} \mathrm{~B}_{\mathrm{VI}}$, представлены в таблице.

Изотропность и малая ширина линий поглощения нонов $\mathrm{Cr}^{3+}$ $\left(\Delta H_{p p}=0,6 \pm 0,1 \Gamma c\right)$, а также наличие в спектре ЭПР только одной линии свидетельствуют о том, что исследуемые ноны расположены в кристаллическом поле строго октаэдрической симметрии. И хотя $\mathrm{Cr}^{3+}$ обладает избыточным по отношению к решетке положительным зарядом, компенсирующий дефект в непосредственной близости иона $\mathrm{Cr}^{3+}$ отсутствует; в противном случае кристаллическое поле расщепило бы основное состояние или, по крайней мере, значительно уширило бы линии поглощения. Компенсация избыточного заряда иона $\mathrm{Cr}^{3+}$ может осуществляться как вакансиями кальция (образцы $\mathrm{CaS}-\mathrm{Cr}$ изготовлены в атмосфере серы), так и одновалентными положительными ионами, например ионами $\mathrm{Na}^{+}$, которые в некотором количестве присутствуют в исходном $\mathrm{CaS}\left[{ }^{10}\right]$. Если учесть, что одна вакансия кальция компенсирует избыточный заряд двух ионов $\mathrm{Cr}^{3+}$, то, естественно, один из двух ионов хрома может и не ассоциироваться с вакансией и, следовательно, половина всех ионов $\mathrm{Cr}^{3+}$ должна быть расположена в кристаллическом поле октаэдрической симметрии. Если же компенсатором заряда служат одновалентные ионы металлов, то следует предположить, что такие дефекты распределены по объему кристалла и удалены от октаэдрических центров хрома на расстояние нескольких постоянных решетки. Аксиальные центры $\mathrm{Cr}^{3+}$ в поликристаллическом $\mathrm{CaS}$ не обнаружены по причине значительной анизотропии спектра ЭПР. Существование таких центров и их свойства в $\mathrm{CaS}$ могут быть установлены при синтезировании монокристаллов CaS-Cr.

\begin{tabular}{|c|c|c|c|c|c|c|c|c|}
\hline Ион & $\begin{array}{l}\text { Мат- } \\
\text { рица }\end{array}$ & $d^{*}, \AA$ & $\begin{array}{c}\text { Сим- } \\
\text { метрия } \\
\text { центра }\end{array}$ & $T, \mathrm{~K}$ & $\begin{array}{c}\Delta H_{p p}, \\
\quad \Gamma c\end{array}$ & $g$ & $\stackrel{A,}{10^{-4}} C M^{-1}$ & Лит-ра \\
\hline $\mathrm{Cr}^{3+}$ & $\begin{array}{l}\mathrm{MgS} \\
\mathrm{CaS} \\
\mathrm{MgO} \\
\mathrm{CaO}\end{array}$ & $\begin{array}{l}2,54 \\
2,83 \\
2,10 \\
2,40\end{array}$ & $O_{h}$ & $\begin{array}{r}298 \\
77 \\
77 \\
298\end{array}$ & $\begin{array}{l}\overline{0} 6 \\
-\end{array}$ & $\begin{array}{l}1,9874 \\
1,9831 \\
1,9800 \\
1,9734\end{array}$ & $\begin{array}{l}15,3 \pm 0,2 \\
15,9 \pm 0,1 \\
16,0 \pm 0,3 \\
16,8 \pm 0,2\end{array}$ & $\begin{array}{l}{\left[{ }^{8}\right]} \\
* * \\
{\left[{ }^{5}\right]} \\
{\left[{ }^{8}\right]}\end{array}$ \\
\hline $\mathrm{Cr}^{+}$ & $\begin{array}{l}\mathrm{MgO} \\
\mathrm{CaS} \\
\mathrm{ZnS} \\
\mathrm{ZnSe} \\
\mathrm{ZnTe} \\
\mathrm{CdTe}\end{array}$ & $\begin{array}{l}2,10 \\
2,83 \\
2,35 \\
2,46 \\
2,63 \\
2,81\end{array}$ & $T_{d}$ & $\begin{array}{c}77 \\
77 \\
77 \\
77 \\
77 \\
4,2\end{array}$ & $\begin{array}{l}0,5 \\
2,3 \\
0,6 \\
- \\
-\end{array}$ & $\begin{array}{l}1,9996 \\
1,9986 \\
1,9995 \\
2,0016 \\
2,0023 \\
1,9997\end{array}$ & $\begin{array}{l}17,6 \pm 0,2 \\
17,4 \pm 0,2 \\
13,4 \pm 0,1 \\
13,3 \pm 0,2 \\
12,4 \pm 0,2 \\
12,781\end{array}$ & $\begin{array}{l}{\left[{ }^{9}\right]} \\
* * \\
{\left[{ }^{2}\right]} \\
{[3]} \\
\left.{ }^{3}\right]\end{array}$ \\
\hline
\end{tabular}

* Данные приведены для $T=298 \mathrm{~K}$, см. $\left[{ }^{11,2}\right]$; ** настоящая работа. Погрешность измерения $g$-фактора $- \pm 0,0005$; погрешность измерения $\Delta H_{p p}- \pm 0,1 \Gamma c$.

Данные, приведенные в таблице, позволяют заключить, что существует корреляция между величиной сверхтонкого магнитного поля на ядре иона ${ }^{53} \mathrm{Cr}^{3+}$ (константой $\left.A\right), g$-фактором и степенью ковалентности связи иона $\mathrm{Cr}^{3+}$ с кристаллической решеткой. На рис. 2 показана зависимость константы $A$ от $g$-фактора иона $\mathrm{Cr}^{3+}$ для различных кубических решеток. В пределах погрешности эксперимента эти параметры парамагнитного иона связаны между собой линейной зависимостью, причем в кристаллах с более значительной степенью ионности константа $A$ принимает бо́льшие значения, a g-фактор - меньшие. Соот- 


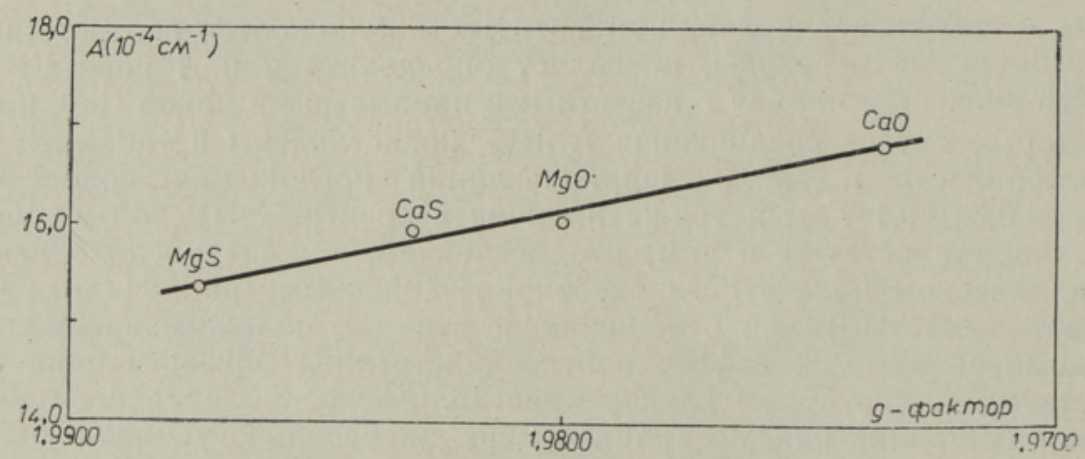

Рис. 2. Зависимость константы $A$ CTC $\left({ }^{53} \mathrm{Cr}^{3+}\right)$ от величины g-фактора иона $\mathrm{Cr}^{3+}$ в кубических кристаллах $\mathrm{A}_{\mathrm{II}} \mathrm{BvI}$.

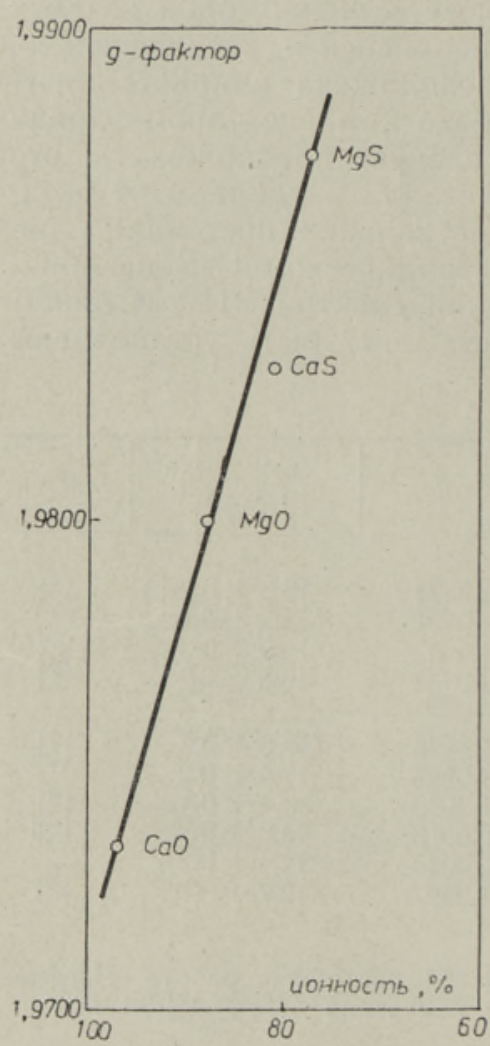

Рис. 3. Зависимость $g$-фактора иона $\mathrm{Cr}^{3+}$ от степени ионности кристалла. ношение между характером связи в кристалле и g-фактором иона $\mathrm{Cr}^{3+}$ показано на рис. 3. Степень ионности связи вычислена по методу Полинга на основе электроотрицательностей образующих кристалл ионов $\left[{ }^{12}\right]$. Для изоморфных кристаллов с общим анионом полученные результаты можно качественно объяснить следующим образом. Сверхтонкое магнитное поле на ядре ${ }^{53} \mathrm{Cr}^{3+}$ возникает, как известно, в результате обменной поляризации замкнутых $1 s^{2}, 2 s^{2}, 3 s^{2}$ оболочек внешними $3 d^{3}$-электронами. Величина индуцированного им контактного поля зависит от взаимного расположения и перекрывания $s$ - и $d$-волновых функций. Увеличение ковалентности связи иона $\mathrm{Cr}^{3+}$ с кристаллической решеткой вызывает частичный перенос неспаренных $d$ электронов к лигандным ионам $\left[{ }^{13}\right]$. Это приводит к уменьшению перекрывания $3 d^{3}$-волновых функций с внутренними $s^{2}$ оболочками и, тем самым, к снижению спиновой плотности на ядре ${ }^{53} \mathrm{Cr}^{3+}$.

В MgO (MgS) расстояние между кристаллообразующими частицами меньше, чем в $\mathrm{CaO}(\mathrm{CaS})$ и, следовательно, перекрывание $3 d^{3}$-волновых функций иона хрома с волновыми функциями лигандных ионов здесь сильнее. Фактически это означает, что связь примесного иона с решеткой кристалла более ковалентна в $\mathrm{MgO}(\mathrm{MgS})$, чем в $\mathrm{CaO}(\mathrm{CaS})$. Можно ожидать, что сверхтонкое взаимодействие иона ${ }^{53} \mathrm{Cr}^{3+}$ в $\mathrm{MgO}$ $(\mathrm{MgS})$ будет меньше, чем в $\mathrm{CaO}(\mathrm{CaS})$. Экспериментальные результаты подтверждают такое предположение. Действительно, при переходе от $\mathrm{MgO}(\mathrm{MgS})$ к $\mathrm{CaO}$ (CaS) константа $A$ иона ${ }^{53} \mathrm{Cr}^{3+}$ увеличивается, свидетельствуя об увеличении $s$-электронной плотности на ядре. На та- 
кую же закономерность указывают данные В. М. Уолша, который, исследуя влияние гидростатического давления на парамагнитные свойства ионов $\mathrm{Cr}^{3+}$ и $\mathrm{Mn}^{2+}$ в кристаллах $\mathrm{MgO}$ и $\mathrm{ZnS}$, установил, что с ростом приложенного к кристаллу давления (т. е. при сжатии кристалла) константа $A$ иона $\mathrm{Mn}^{2+}\left(3 \dot{d}^{5}\right)$ линейно уменьшается, a $g$-фактор иона $\mathrm{Cr}^{3+}$ увеличивается $\left[{ }^{14}\right]$.

Увеличение g-фактора иона $\mathrm{Cr}^{3+}$ при сокращении расстояния до лигандных ионов вызвано уменьшением орбитального вклада от вышележащего триплета ${ }^{4} T_{2 g}$, а также более широким энергетическим зазором между основным $\left({ }^{4} A_{2 g}\right)$ и возбужденным $\left({ }^{4} T_{2 g}\right)$ состояниями иона.

Заканчивая обсуждение влияния кристаллической решетки на магнитные свойства иона $\mathrm{Cr}^{3+}$, заметим, что наличие линейной зависимости между величинами g-фактора и константы $A$ иона $\mathrm{Cr}^{3+}$ в различных матрицах указывает на однотипность структуры центров $\mathrm{Cr}^{3+}$ в этих матрицах и является дополнительным подтверждением интерпретации спектра ӘПР ионов $\mathrm{Cr}^{3+}$ в $\mathrm{CaS}$.

Ионы $\mathrm{Cr}^{+}$в CaS. Наряду с ионами $\mathrm{Cr}^{3+}$ в $\mathrm{CaS}$ обнаружено состояние $\mathrm{Cr}^{+}$. Одновалентный хром $\left(3 d^{5},{ }^{6} S_{5 / 2}\right)$ возникает при облучении рентгеновскими лучами образцов $\mathrm{CaS}-\mathrm{Cr}$, которые после окончания синтеза были подвергнуты быстрому охлаждению до комнатной температуры. Спектр ЭПР ионов $\mathrm{Cr}^{+}$в $\mathrm{CaS}$ аналогичен спектру ионов $\mathrm{Cr}^{3+}$, он состоит также из центральной линии с $g=1,9986 \pm 0,0005$ и четырех равноотстоящих линий СТС с расщеплением $\Delta H=1 \overline{8,6} \pm 0,4 \Gamma c$. Отношение интенсивности центральной линии к интенсивностям линий СТС равно $1 / 45 \pm 5 \%$, что близко к теоретическому значению. Величины g-факторов ионов $\mathrm{Cr}^{+}$в $\mathrm{CaS}$ и $\mathrm{MgO}$ приблизительно одинаковы и отличаются от чисто спинового значения 2,0023 незначительно. Последнее обстоятельство подтверждает правильность идентификации описанного выше спектра, так как малое смещение g-фактора является характерной особенностью всех ионов в ${ }^{6} S_{5} / 2$-состоянии. Наблюдаемый спектр ионов $\mathrm{Cr}^{+}$обусловлен переходами между подуровнями с $m_{S}=$ $\pm 1 / 2$. Переходы $( \pm 3 / 2 \leftrightarrow \pm 1 / 2)$ и $( \pm 5 / 2 \leftrightarrow \pm 3 / 2)$ из-за сильной угловой зависимости в порошке не проявляются.

Существуют и другие аналогии в магнитных свойствах ионов $\mathrm{Cr}^{+}$ в $\mathrm{CaS}$ и MgO. Tак, в $\mathrm{CaS}$ величина сверхтонкого взаимодействия больше для $\mathrm{Cr}^{+}$, чем для $\mathrm{Cr}^{3+}$ (см. таблицу). Подобное увеличение константы $A$ наблюдается в $\mathrm{MgO}$ при переходе от $\mathrm{Cr}^{3+} \mathrm{K} \mathrm{Cr}^{+}$, а также от $\mathrm{Mn}^{4+}\left(3 d^{3} ; A=70,8 \cdot 10^{-4} \quad \mathrm{CM}^{-1}\right) \quad \mathrm{K}$ $\mathrm{Mn}^{2+}\left(3 d^{5} ; A=81,5 \cdot 10^{-4} \mathrm{Cm}^{-1}\right)$. Это объясняется тем, что в ионах с конфигурацией $3 d^{5}$ поляризация внутренних $s$-оболочек валентными $d$ электронами сильнее, чем в ионах с конфигурацией $3 d^{3}$, а следовательно, и плотность $s$-электронов на ядре больше.

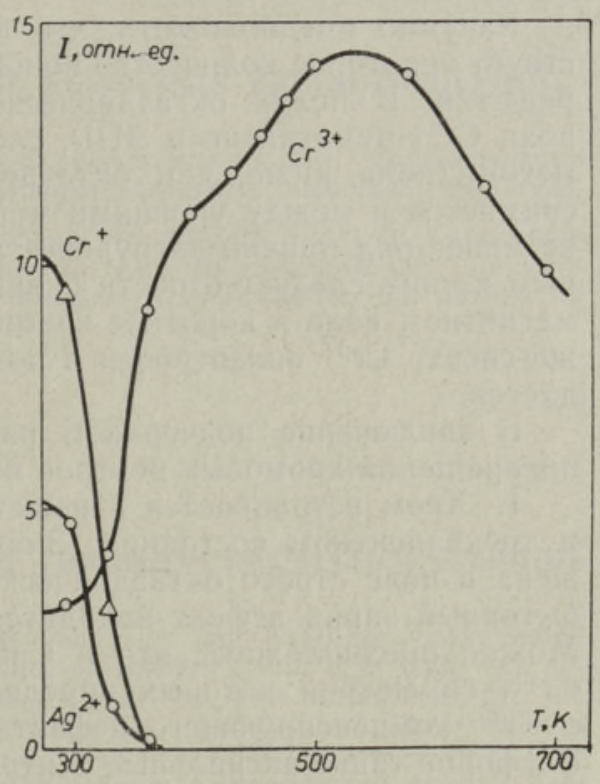

Рис. 4. Кривые неизотермической релаксации парамагнитных центров в закаленном фосфоре $\mathrm{CaS}-\mathrm{Cr}$, Ag. Фосфор возбужден рентгеновскими лучами при $293 \mathrm{~K}$. 
Ионы $\mathrm{Cr}^{+}$в $\mathrm{CaS}$, также как и в $\mathrm{MgO}$, возникают в процессе облучения ионизирующей радиащией. На рис. 4 показаны кривые неизотермической релаксации хромовых центров в $\mathrm{CaS}$, активированном ионами хрома и серебра. Спектр ЭПР такого фосфора изображен на рис. 1 , б. Серебро входит в $\mathrm{CaS}$ в виде одновалентных ионов, замещающих ионы $\mathrm{Ca}^{2+}$ в регулярных узлах решетки $\left[{ }^{15}\right]$. В процессе рентгеновского облучения ион $\mathrm{Ag}^{+}$захватывает дырку и переходит в $\mathrm{Ag}^{2+}$, который дает характерный сигнал ЭПР (рис. 1, б). Термическое разрушение $\mathrm{Ag}^{2+}$ в области температур 290-370 K связано с освобождением дырок с $\mathrm{Ag}^{2+}$ и их рекомбинацией на ионе $\mathrm{Cr}^{+}$. Как видно из рис. 4, разрушение ионов $\mathrm{Ag}^{2+}$ сопровождается исчезновением ионов $\mathrm{Cr}^{+}$и образованием ионов $\mathrm{Cr}^{3+}$. Эти процессы можно выразить уравнениями:

$$
\begin{aligned}
& \mathrm{Ag}^{2+} \rightarrow \mathrm{Ag}^{+}+\mathrm{fe}^{+} \\
& \mathrm{Cr}^{+}+e^{+} \rightarrow \mathrm{Cr}^{2+} \\
& \mathrm{Cr}^{2+}+e^{+} \rightarrow \mathrm{Cr}^{3+} .
\end{aligned}
$$

Дальнейшее увеличение числа $\mathrm{Cr}^{3+}$ обязано освобождению дырок с ловушек неизвестной природы. Спад на кривой при $T \geqslant 530 \mathrm{~K}$ обусловлен, очевидно, термической ионизацией $\mathrm{Cr}^{3+}$.

Тот факт, что $\mathrm{Cr}^{+}$образуется только в закаленных образцах $\mathrm{CaS}-\mathrm{Cr}$, может быть объяснен наличием в таких фосфорах эффективных ловушек для дырок. В этом случае электроны при возбуждении фосфора захватываются ионами $\mathrm{Cr}^{2+}$ без последующей рекомбинации с дырками.

Значительная ширина линий ЭПР ионов $\mathrm{Cr}^{+}$в $\mathrm{CaS}$ при $77 \mathrm{~K}$ (см. таблицу и рис. 1, б) связана, вероятно, с анизотропией перехода $(-1 / 2 \leftrightarrow+1 / 2)$, имеющей. порядок $a^{2} / g^{2} \beta^{2} H_{0}$, где $a-$ константа тонкого расщепления и $H_{0}$ - величина магнитного поля для резонансной линии $\mathrm{Cr}^{+}$.

Разумно предположить, что в $\mathrm{CaS}$ наряду с центрами $\mathrm{Cr}^{3+}$ существует некоторое количество ионов $\mathrm{Cr}^{2+}$, замещающих ионы $\mathrm{Ca}^{2+}$ в узлах решетки. В полях октаэдрической симметрии основное состояние ${ }^{2} E$ иона $\mathrm{Cr}^{2+}$ немагнитно и ЭПР, следовательно, не наблюдаем. Если симметрия поля ниже, чем октаэдрическая, то орбитальное вырождение снимается и между уровнями могут быть индуцированы переходы. Тем не менее ряд причин затрудняет наблюдение ЭПР ионов $\mathrm{Cr}^{2+}$. $\mathrm{K}$ основным из них следует отнести большую величину расщепления в нулевом магнитном поле и короткое время спин-решеточной релаксации. $\mathrm{K}$ тому же спектр $\mathrm{Cr}^{2+}$ анизотропен и поэтому регистрации в порошках не поддается.

В заключение подчеркнем ряд аналогий в структуре и процессах превращений хромовых центров в $\mathrm{CaS}$ и $\mathrm{MgO}$.

1. Хром встраивается в решетки этих кристаллов преимущественно в трехвалентном состоянии. Значительная часть ионов $\mathrm{Cr}^{3+}$ расположена в поле строго октаэдрической симметрии, и компенсирующий избыточный заряд дефект находится от них на значительном расстоянии. Можно предположить, что в $\mathrm{CaS}$, как и в $\mathrm{MgO}$, существуют центры $\mathrm{Cr}^{3+}$, симметрия которых определяется положением ассоциированного с $\mathrm{Cr}^{3+}$ компенсирующего дефекта. В спектрах ЭПР порошкообразных фосфоров такие аксиальные центры не наблюдаются.

2. При облучении кристаллов рентгеновскими лучами трехвалентный ион хрома захватывает электрон и переходит в $\mathrm{Cr}^{2+}$. Последующая термическая обработка кристаллов вновь восстанавливает $\mathrm{Cr}^{3+}$-центры (cм. $\left.\left[{ }^{5}\right]\right)$.

3. Одновалентный хром может возникать в этих матрицах при низ- 
котемпературном возбуждении кристаллов ионизирующим излучением. Термическая стабильность ионов $\mathrm{Cr}^{+}$сравнительно невелика (см. рис. 4 и $\left.\left[{ }^{9}\right]\right)$. Симметрия центров $\mathrm{Cr}^{+}-$октаэдрическая.

Авторы благодарят И. Яэка за руководство настоящей работой, а также Т. Партс и К. Реало за приготовление объектов.

\section{Л И Т Е Р А Т У Р А}

1. Vallin, J. T., Watk ins, G. D., Phys. Rev. (B), 9, 2051 (1974).

2. Titlle, R. S., Phys. Rev., 131, 623 (1963).

3. Title, R. S., Phys. Rev., 133, A 1613 (1964).

4. L u d w i g, G. W., L o r e n z, M. R., Phys. Rev., 131, 601 (1963).

5. L o w, W., Phys. Rev., 105, 801 (1957).

6. Low, W., Rubins, R. S., Paramagnetic Resonance, 1, Jerusalem Conf. (Ed. by W. Low), New York, 1963, p. 79.

7. We rt z, J. E., A u z in s, P., Phys. Rev., 106, 484 (1957).

8. Auz ins, P., Orto n, J. W., Wertz, J. E., Paramagnetic Resonance, 1, Jerusalem Conf. (Ed. by W. Low), New York, 1963, p. 90.

9. Rius, G., Herve, A., Solid State Comm., 11, 795 (1972).

10. Я э к И. В., Х ю т т Г. И., Изв. АН СССР, Сер. физ., 35, 1505 (1971).

11. P a uling, L., The Nature of the Chemical Bond, 3 ed., Ithaca - New York, 1960.

12. Philli ps, J. C., Rev. Modern Phys., 42, 317 (1970).

13. Owe n, J., Thorniley, J. H. M., Rept. Progr. Phys., 29, 675 (1966).

14. W a $1 \mathrm{sh}$, W. M., Phys. Rev., 122, 762 (1961).

15. К узнецов А. С., Хютт Г. И., Яэк И. В., Тр. ИФ АН ЭССР, 43, 223 (1975).

\section{Тартуский государственный университет}

Поступила в редакцию $21 /$ II 1977

\section{SEEMAN, A. KUZNETSOV}

\section{$\mathrm{Cr}^{3+-} \mathrm{JA} \mathrm{Cr}^{+- \text {-IOONIDE PARAMAGNETILINE RESONANTS KALTSIUMSULFIIDIS }}$}

On uuritud $\mathrm{Cr}^{3+-}$ ja $\mathrm{Cr}^{+-}$-lisandioonide spektreid polükristalses $\mathrm{CaS}$-s. Neeldumisjoonte kuju, nende väike laius ning põhinivoo ${ }^{4} F_{\% / 2}$ esialgse lōhenemise puudumine tōendavad, et $\mathrm{Cr}^{3+}$-ioonid asetsevad oktaeedrilise sümmeetriaga elektriväljas. $\mathrm{Cr}^{3+}$ - ja $\mathrm{Cr}^{+}$-ioonide $g$-faktorid ning ülipeeninteraktsiooni konstantide väärtused on lähedased $\mathrm{Cr}^{3+} \cdot$ ja $\mathrm{Cr}^{+}$. ioonide vastavatele parameetritele mōningates teistes $\mathrm{A}_{I I} \mathrm{~B}_{\mathrm{V}_{I}}$ ühendites. On uuritud ka $\mathrm{Cr}^{3+}$. ja $\mathrm{Cr}^{+}$-ioonide termilist stabiilsust.

\section{SEEMAN, A. KUZNETSOV}

\section{ELECTRON PARAMAGNETIC RESONANCE OF CHROMIUM IN CALCIUM SULPHIDE}

The paramagnetic resonance spectra of the $\mathrm{Cr}^{3+}$ and $\mathrm{Cr}^{+}$ions have been investigated in the powder of $\mathrm{CaS}$. The paramagnetic resonance parameters $g$ and $A$ of both ions are determined and compared to the corresponding values of these parameters for the $\mathrm{Cr}^{3+}$ and $\mathrm{Cr}^{+}$ions in some other $\mathrm{A}_{I I} \mathrm{~B}_{\mathrm{VI}}$ compounds. It is found that a linear relation exists between the values of $g$ and $A$ for the $\mathrm{Cr}^{3+}$ ions in cubic lattices $\mathrm{A}_{I I} \mathrm{~B}_{\mathrm{VI}}$. The narrow absorption line of the $\mathrm{Cr}^{3+}$ ions and the failure to observe any ground state splitting indicate that the $\mathrm{Cr}^{3+}$ ions dispose in a pure octahedral field. The thermal stability of the chrome ions has also been dealt with. 